\title{
Illusory temporal order for stimuli at different depth positions
}

\author{
MaKoto ICHIKAWA \\ Chiba University, Inage, Chiba, Japan
}

\begin{abstract}
We used four experiments to examine how the perceived temporal order of two visual stimuli depends on the depth position of the stimuli specified by a binocular disparity cue. When two stimuli were presented simultaneously at different depth positions in front of or around a fixation point, the observer perceived the more distant stimulus before the nearer stimulus (Experiments 1 and 2). This illusory temporal order was found only for sudden stimulus presentation (Experiment 3 ). These results suggest that a common processing, which is triggered by sudden luminance change, underlies this illusion. The strength of the illusion increased with the disparity gradient and the disparity size (Experiment 4). We propose that this illusion has a basis in the processing of motion in depth, which would alert the observer to a potential collision with an object that suddenly emerges in front of the observer.
\end{abstract}

The perception of a temporal relationship for events in visual space depends on various factors related to the physical properties of stimuli and to the observer's conscious state. For example, visual latency for stimulus presentations depends on the luminance level of the stimuli (see, e.g., Rogers \& Anstis, 1972). Apparent relative velocities for moving stimuli are affected by contrasts between the stimuli and the background (see, e.g., Anstis, 2001). The perception of visual simultaneity for two stimuli also depends on the eccentricity of the stimulus presentation (see, e.g., Ichikawa, 2002; Mitrani, Shekerdjiiski, \& Yakimoff, 1986). Simultaneous changes in different submodalities of the visual system, such as color and motion (see, e.g., Arnold, Clifford, \& Wenderoth, 2001; Aymoz \& Viviani, 2004; Bedell, Chung, Ogmen, \& Patel, 2003; Linares \& López-Moliner, 2006; Moutoussis \& Zeki, 1997a, 1997b; Nishida \& Johnston, 2002; Viviani \& Aymoz, 2001) and color and location (see, e.g., Pisella, Arzi, \& Rossetti, 1998; Tanaka \& Shimojo, 1996), are perceived as asynchronous events. Visual attention can also modulate processing around the attended area and, consequently, the temporal order and simultaneity for presentations of stimuli in attended and nonattended areas of the visual field (see, e.g., Hikosaka, Miyauchi, \& Shimojo, 1993; Posner, Snyder, \& Davidson, 1980; Shimojo, Miyauchi, \& Hikosaka, 1997; Stelmach \& Herdman, 1991). Studies examining the illusory flash-lag effect have shown that a continuous change in position (see, e.g., Bachmann, Luiga, Põder, \& Kalev, 2003; Brenner \& Smeets, 2000; Eagleman \& Sejnowski, 2000; Krekelberg \& Lappe, 2001; Nijhawan, 1994; Purushothaman, Patel, Bedell, \& Ogmen, 1998; Whitney \& Murakami, 1998) and in various other visual dimensions, such as luminance and color (see, e.g.,
Sheth, Nijhawan, \& Shimojo, 2000), introduces temporal differences in the processing of the changing stimulus and flash. This illusory flash-lag effect can be enhanced by distracting attention from the stimulus (see, e.g., Baldo, Kihara, Namba, \& Klein, 2002; Murakami, 2001; Shioiri, Yamamoto, \& Yaguchi, 2002); it is reduced significantly by active observation (see, e.g., López-Moliner \& Linares, 2006) or by a subjective mental set in which the observer controls the stimulus change (see, e.g., Ichikawa \& Masakura, 2004, 2006). Current psychophysical studies are examining whether other factors can be added to the list of factors that affect the temporal relationship for events in visual space.

In the present study, we examined whether depth positions of the stimuli specified by a binocular disparity cue could be added to the list described above. Earlier studies using a single visual stimulus with a fixation point have demonstrated that processing of a stimulus nearer than the fixation plane is more rapid than that of a stimulus that is more distant than the fixation plane. For example, the latency of a converging eye movement is shorter than that of a diverging one if the angle of the convergence/divergence is smaller than $20^{\circ}$ (see, e.g., Alvarez, Semmlow, \& Pedrono, 2005; Hung, Zhu, \& Ciuffreda, 1997; Yang, Bucci, \& Kapoula, 2002). The latency of depth perception for crossed disparity, which specifies stimulus position in a space nearer than the fixation plane, is shorter than that for uncrossed disparity, which specifies stimulus position in a space that is farther than the fixation plane (see, e.g., Bradshaw, Rogers, \& De Bruyn, 1995; Patterson et al., 1995), in particular when there are no monocular zones in the stimulus (see, e.g., Becker, Bowd, Shorter, King, \& Patterson, 1999). Several studies have demonstrated

M. Ichikawa, ichikawa@I.chiba-u.ac.jp 
that stereoacuity is better for crossed disparity than for uncrossed disparity (e.g., Landers \& Cormack, 1997; Woo $\&$ Sillanpaa, 1979). Howard and Rogers $(1995,2002)$ and Mustillo (1985) have shown that depth from crossed disparities is identified or discriminated more rapidly than that from uncrossed disparities. In addition, several studies have examined a binocular stimulus in a real space (see, e.g., Downing \& Pinker, 1985; Gawryszewski, Riggio, Rizzolatti, \& Umiltà, 1987; Miura, Shinohara, \& Kanda, 2002), showing that a shift of visual attention from a fixation point toward a nearer stimulus is faster than a shift toward a more distant stimulus. Considering these previous studies using a single stimulus with a fixation point, one might predict that a stimulus presented in a space nearer than the fixated plane would be perceived more rapidly than if it were presented in a space more distant than the fixation plane, even if both stimuli were presented simultaneously.

In Experiment 1, we examined the degree to which the depth positions of visual stimuli affect the perceived temporal order of the presentations of two stimuli at different depth positions defined by a binocular disparity cue with various stimulus onset asynchronies (SOAs). Observers judged the temporal order of the presentations of two stimuli. The results showed effects of depth position on the apparent temporal order of the stimulus presentations. That is, the observer perceived that the more distant stimulus appeared before the nearer one. This result cannot be explained merely by the rapid processing for the crossed disparity, as has been reported in previous studies using a single stimulus. In our other experiments, we examined the effects of stimuli's depth position on temporal order in visual space. Finally, we discuss the implications of the spatiotemporal characteristics of the visual experience.

\section{EXPERIMENT 1}

In the first experiment, two stimuli were presented at different depth positions and with various SOAs. In each trial, observers were asked to judge the temporal order of the presentations of the stimuli.

\section{Method}

Stimuli and Apparatus. As is shown in Figure 1A, two mirrors $(10.0 \times 12.5 \mathrm{~cm})$ and two 15 -in. displays (Mitsubishi RD15 MII, $60 \mathrm{~Hz}$ ) controlled by an Apple Macintosh IIfx (with two 8/24 Apple video cards) were arranged as a haploscope to present binocular disparity cues. The total viewing distance to each display was $45 \mathrm{~cm}$ $(10+35 \mathrm{~cm})$.

A red square $\left(23.8 \times 23.2 \operatorname{arc} \min , 37.5 \mathrm{~cd} / \mathrm{m}^{2}\right)$ was presented as a fixation point on the surface of the display with zero disparity (Figure 1B). Two white stimuli $\left(20.8 \times 2.9 \mathrm{arc} \min , 118.5 \mathrm{~cd} / \mathrm{m}^{2}\right)$ were presented 56.5 arc min above the fixation point on a gray background $\left(76.5 \mathrm{~cd} / \mathrm{m}^{2}\right)$. The distance between the two stimuli was 2.9 arc min for the left (or right) eye and 8.7 arc min for the right (or left) eye, giving a 5.8 arc min binocular disparity cue. The disparity gradient for the two stimuli, which is defined as the rate between the binocular discrepancy and the distance between the stimuli, was kept at $2 / 3$, because, if it were greater than 2.0 , the observer would be expected to have difficulty detecting depth when viewing the two stimuli at different depth positions (Howard \& Rogers, 1995, 2002). The disparity cue specifies that the left (or right) stimulus is nearer the observer than the other stimulus if the distance between the two stimuli for the left (or right) eye image is narrower than that for the right (or left) eye image (Figure 1C). It was confirmed that these distances between the stimuli on each half image had no significant effect on the apparent temporal order of the stimuli if the binocular disparity was zero for the two stimuli (Ichikawa, 2002).

Five conditions were used for the depth positions of the stimuli, specified by the binocular disparity cue between the stimuli and the fixation plane (Figure 1C). In preliminary experiments, we used other depth position conditions, in which the stimuli were more distant from the fixation plane. In those conditions, some observers reported double vision for the stimuli. They had difficulty achieving consistent depth perception. For that reason, we used the present five depth position conditions, for which no observer reported problems related to viewing the depth of the stimuli.

Procedure. The two stimuli were presented on different depth planes. The relative depth between the stimuli was always specified by 5.8 arc min of binocular disparity. Seven SOA conditions were used for presenting the two stimuli, ranging from -48 to $+48 \mathrm{msec}$ in 16-msec increments (a positive value of SOA indicates that the presentation of the nearer stimulus was earlier than that of the more distant stimulus).

Ten blocks were used for each observer. In each block, there were 70 stimulus conditions, presented in random order: 7 SOAs $\times$ 2 depth orders (left or right stimulus nearer the observer) $\times 5$ depth positions of the stimuli. Consequently, each stimulus condition was presented 10 times to each observer.

Each experimental trial began with presentation of the fixation point; the observer pressed a key on the keyboard to start the trial (Figure 2). Following a random interval of 2,000-2,256 msec after the observer had pressed the key, the first stimulus was presented. After the second stimulus was presented at one of the seven SOAs, the observer pressed a key to report whether the nearer or the more distant stimulus had been perceived later. The stimuli disappeared when the observer pressed the key. To familiarize themselves with the equipment and procedure, observers had a training session with at least 20 trials before the experimental trials. Sessions for each observer lasted about $60 \mathrm{~min}$, including the training sessions.

Observers. The author and 5 naive graduate and undergraduate students served as observers ( 2 female and 3 male). Their ages ranged from 20 to 36 years. All 6 observers had normal or correctedto-normal visual acuity. In a preliminary test that used the same stimuli as those in Experiment 1, all observers had correct depth order perception for the two stimuli in almost all observations (at least $95 \%$ of the observations).

\section{Results and Discussion}

We found no consistent effect of depth order condition (whether the left or the right stimulus was perceived as nearer the observer). Therefore, the results of this condition were combined in the following analyses.

Figure $3 \mathrm{~A}$ shows the frequency of the trials in which the observer perceived that the presentation of the nearer stimulus followed the presentation of the more distant stimulus, for 2 observers. Even when the two stimuli were presented simultaneously, observers tended to perceive that the presentation of one stimulus followed that of the other in many of the depth position conditions. This result suggests that the temporal order of stimulus presentation in visual space depends on the depth relationship between the stimuli.

To obtain the SOA for the simultaneous perception of the presentation of the two stimuli, a probit analysis (see Finney, 1971) was conducted for the individual observers' data for each depth position condition. Figure 3B shows 
A
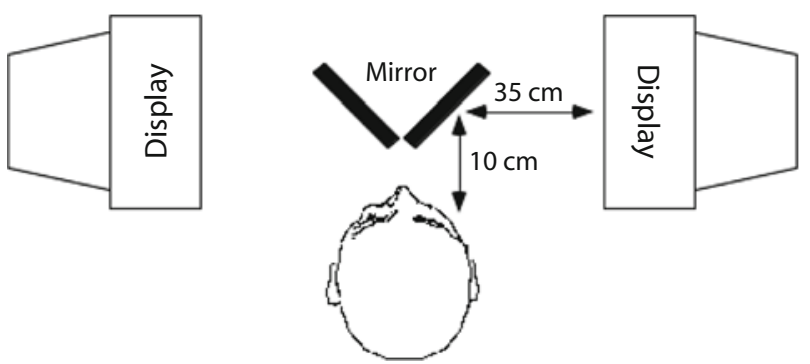

B
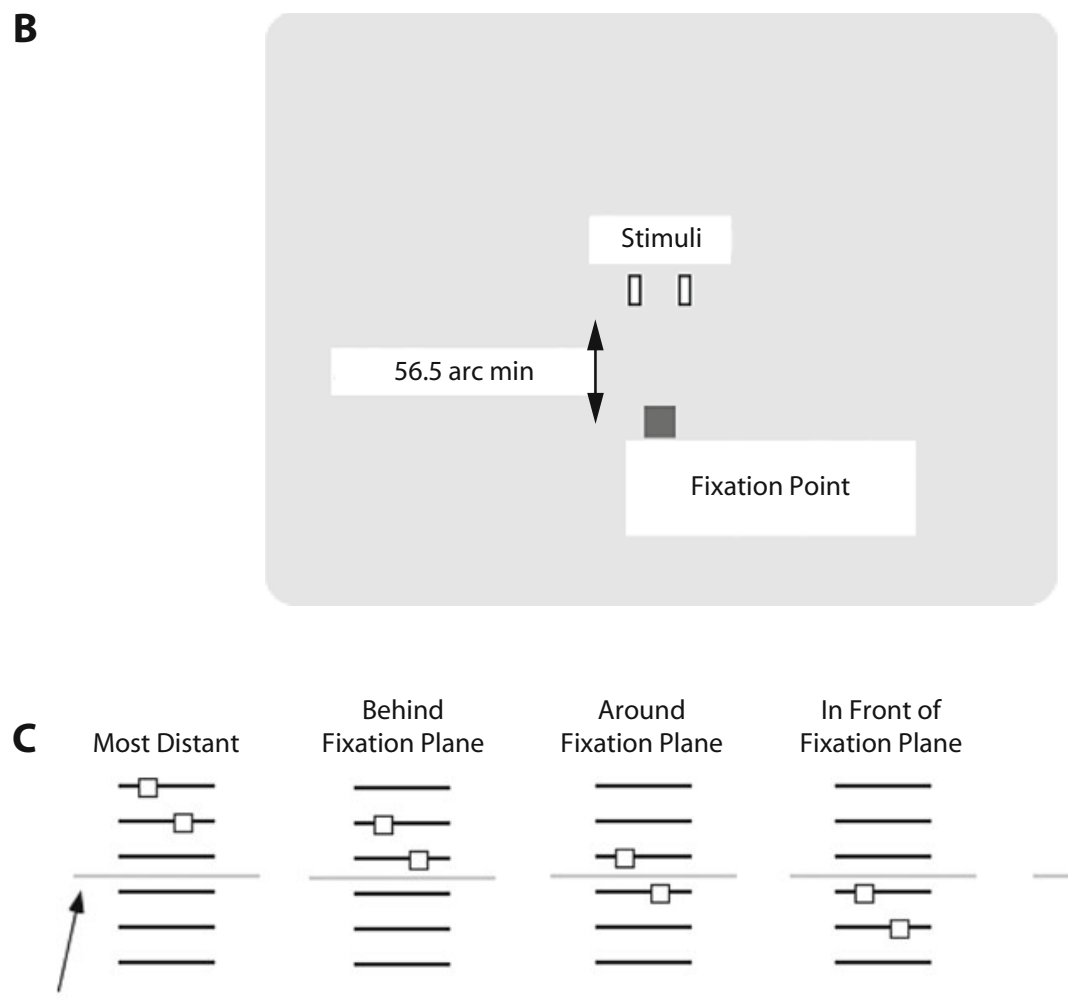

Behind

Around
Fixation Plane

In Front of

Fixation Plane
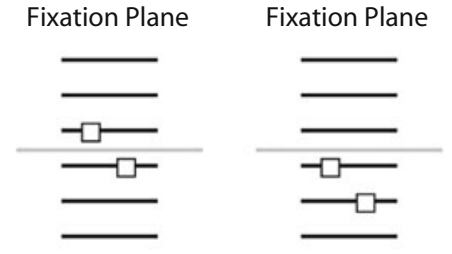

Nearest

Fixation

Plane
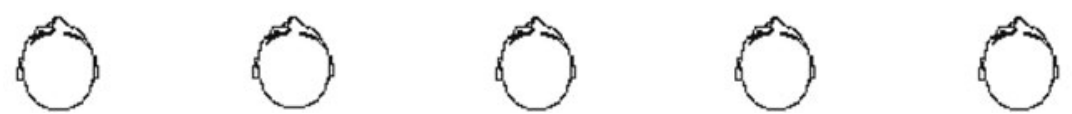

Figure 1. Stimulus presentation in Experiment 1. (A) Equipment for presenting a binocular disparity cue. Two displays were arranged as a haploscope. (B) Stimulus configuration. Two white stimuli were presented above a red fixation point on a gray background. (C) Five conditions for the depth positions of the two stimuli. There were six depth planes defined by binocular disparity relative to the fixation plane (uncrossed, 14.5 arc min; uncrossed, 8.7 arc min; uncrossed, 2.9 arc min; crossed, 2.9 arc min; crossed, 8.7 arc min; and crossed, 14.5 arc min). The two stimuli were presented on the two adjacent depth planes. This diagram shows the case in which the right stimulus is relatively nearer than the other stimulus.

the means of the SOAs at which, in $50 \%$ of the trials, the observer perceived the presentation of the nearer stimulus as following the presentation of the more distant stimulus for each condition (a positive value of the SOA indicates that the presentation of the nearer stimulus was earlier than that of the more distant stimulus). In Figure 3B, the means and the $t$ statistic $(p<.05)$ in terms of $95 \%$ confidence limits suggest that the SOAs were significantly greater than zero in the around-fixation-plane condition, in-front-of-fixation-plane condition, and nearest condition, whereas the SOAs in the most distant condition and behind-fixation-plane condition were not significantly different from zero. These results indicate that, when at least one stimulus was presented in the space in front of the fixation plane, observers perceived that the more distant stimulus appeared before the nearer stimulus. This tendency was opposite to the prediction of rapid processing for a single stimulus nearer than the fixation plane, 
Keypress

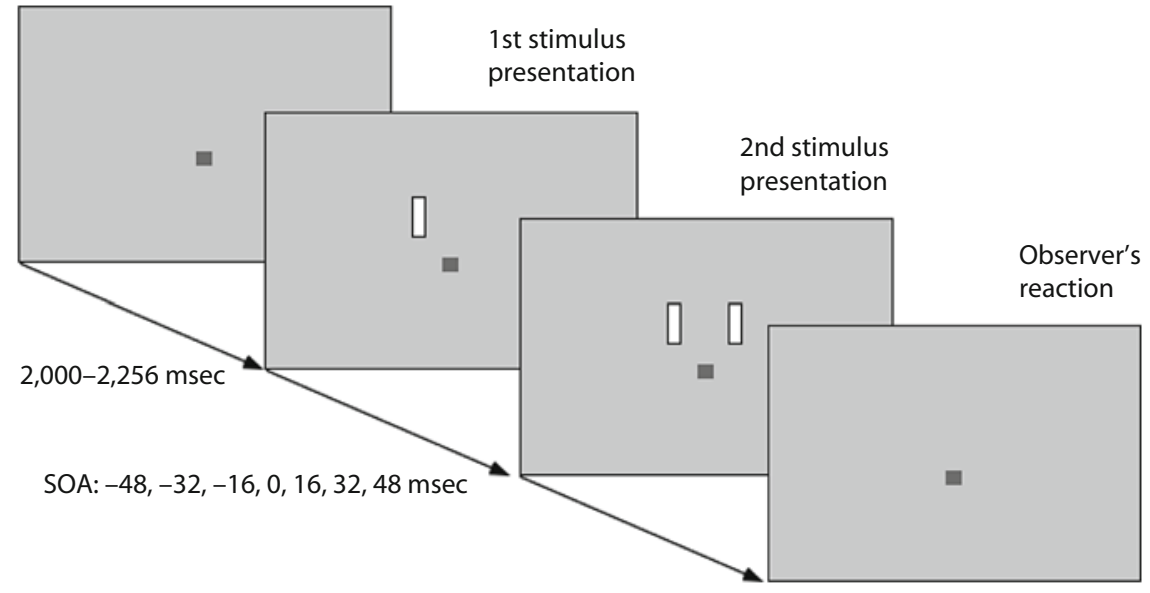

Figure 2. Sequence of the stimulus presentation in Experiment 1. This diagram shows the case in which the presentation of the right stimulus follows the presentation of the left stimulus.

which has been reported in previous studies (e.g., Howard \& Rogers, 1995, 2002; Mustillo, 1985).

As Figure 3B shows, the visual system might require a negative SOA for visual simultaneity in the most distant condition, in which both stimuli were presented in the space beyond the fixation plane. However, the absolute value of this negative SOA was less than the positive SOA for the stimuli presented in the space in front of the fixation plane. These results imply anisotropy in the illusory temporal bias in terms of the relative depth of the stimuli, as specified by the binocular disparity cue. That is, the illusory temporal bias for the space in front of the fixation plane is greater than that for the space beyond the fixation plane.

We conducted a one-way repeated measures ANOVA for the condition of depth position, using the SOA data from the 6 observers (Figure $3 \mathrm{~B}$ ). The main effect of condition was significant $[F(4,16)=14.504, p<.001]$. Tukey's post hoc tests showed that the SOA in the most distant condition was significantly smaller than those in the aroundfixation-plane, in-front-of-fixation-plane, and nearest conditions, and that the SOA was significantly smaller in the behind-fixation-plane condition than in the in-front-offixation-plane and nearest conditions $(p<.05)$.

\section{EXPERIMENT 2}

The results of Experiment 1 demonstrated the illusory temporal bias associated with the depth position of the stimuli. That is, when two stimuli were simultaneously presented at different depth positions around or in front of the fixation point, the observers tended to perceive that the more distant stimulus appeared before the nearer stimulus. In addition, when the stimuli were presented at different depth positions more distant than the fixation point, observers showed a tendency to perceive the stimulus that was more distant as following after the presentation of the nearer stimulus. In the second experiment, we examined whether these results of Experiment 1 depended on inaccurate fixation and mismatch of the horizontal stimuli.

One might wonder whether inaccurate fixation caused the positive and large SOAs for the stimuli presented at depth positions around or in front of fixation. For instance, the possibility exists that observers fixated a depth plane that was more distant than the fixation plane because of underconvergence, and that the processing time increased with the increment in the distance between the stimulus and the fixated plane. The effect of fixation disparity was tested in order to examine the notion that inaccurate fixation caused the positive and large SOAs for the stimuli presented at depth positions around the fixation point.

Because the stimuli were placed horizontally in the first experiment, the retinal image of a stimulus in the right eye might be mismatched with the retinal image of the other stimulus in the left eye by pairing the nearest neighbors. This mismatch might contaminate the data. Stimulus images were separated vertically in Experiment 2 to avoid such a mismatch.

\section{Method}

Stimuli and Apparatus. We used the same apparatus as in Experiment 1 . We used a red nonius fixation. A T-shape and an inverted T-shape $(14.9 \times 29.0$ arc min $)$ were presented to the left and right eyes, respectively, with zero disparity at the center of the display; they appeared as a red cross $\left(29.8 \times 29.0 \operatorname{arc~} \mathrm{min}, 37.5 \mathrm{~cd} / \mathrm{m}^{2}\right)$ when fused correctly and aligned vertically. Two white stimuli $(20.8 \times$ $2.9 \operatorname{arc} \mathrm{min}, 118.5 \mathrm{~cd} / \mathrm{m}^{2}$ ) were presented on the right side of the fixation cross. The zero disparity point between the stimuli was fixated at 58 arc min right of the center of the fixation cross (Figure 4). To achieve 5.8 arc min of binocular disparity, the horizontal distance between the two stimuli was 2.9 arc min for the left (or right) eye and 8.7 arc min for the right (or left) eye, as in Experiment 1. Although the size of the white stimuli was equal to that used in Experiment 1, they were lined up vertically. The vertical gap between the stimuli was 5.3 arc min. As in Experiment 1, there were five stimulus depth positions, as specified by the binocular disparity cue between the stimuli and the fixation plane (Figure 1C).

Procedure. Nine time lags were used between the presentation of the target stimulus and the luminance change in the background, 

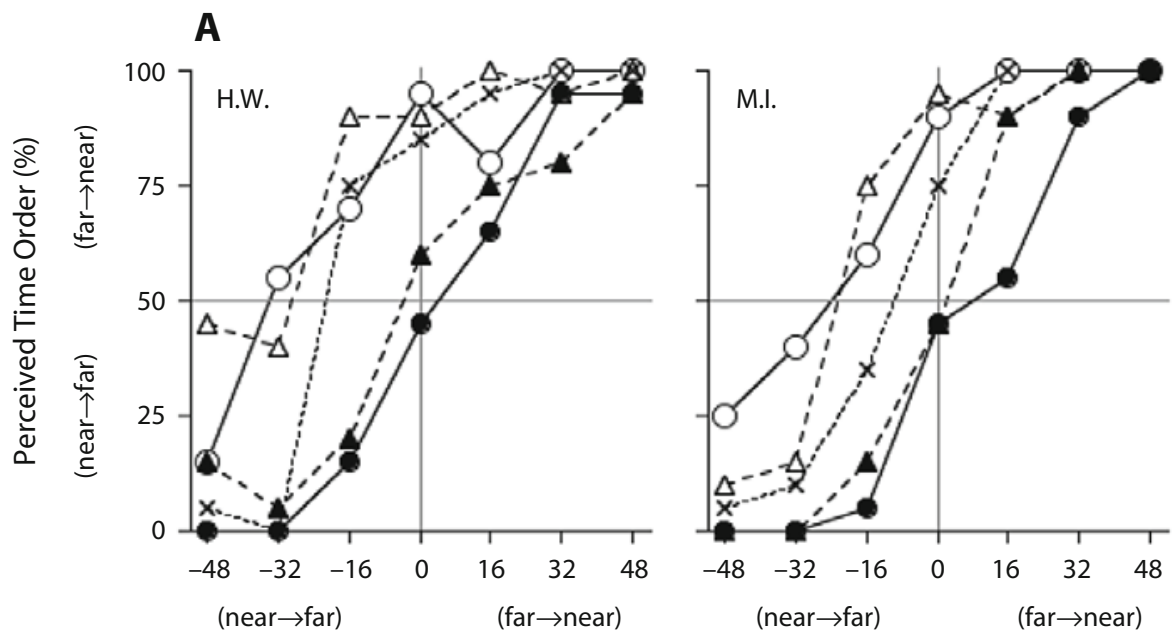

Depth Position Conditions

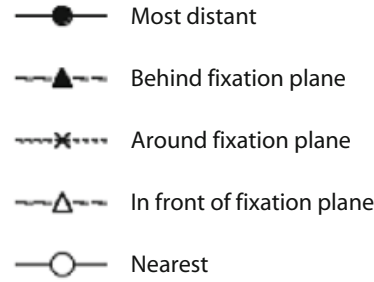

SOA (msec)

B

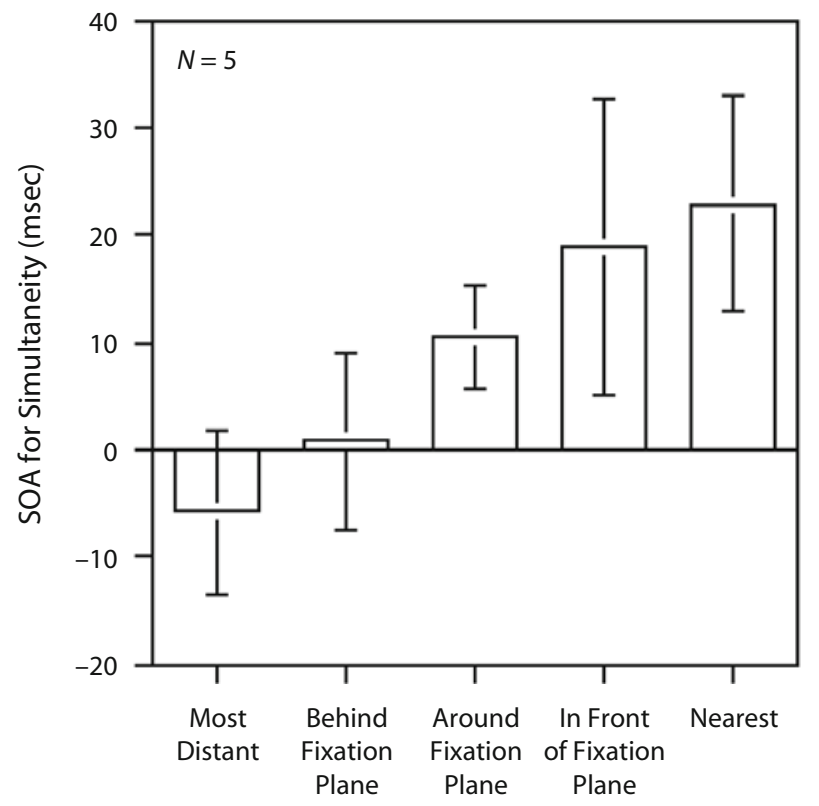

Depth Position Conditions

Figure 3. Results of Experiment 1. (A) Examples for 2 observers (H.W. and M.I.). The frequency at which the presentation of the relatively nearer stimulus was perceived to follow that of the more distant stimulus for each depth position condition. (B) The mean and $\mathbf{9 5 \%}$ confidence limit of the stimulus onset asynchrony (SOA) at which the two stimuli appeared to be presented at the same time in each of the depth position conditions. Positive (or negative) values indicate that the presentation of the relatively nearer stimulus should precede (or follow) the presentation of the more distant one to achieve apparent simultaneity for the presentations of the two stimuli.

ranging from -64 to $+64 \mathrm{msec}$ in $16-\mathrm{msec}$ increments (a positive value of the SOA indicates that the presentation of the nearer stimulus was earlier than that of the more distant stimulus). Five blocks were used for each observer. In each block, there were 90 stimulus conditions, presented twice in random order: 9 SOAs $\times 2$ depth orders (upper or lower stimulus nearer the observer) $\times 5$ depth positions of the stimuli. Therefore, each stimulus condition was presented 10 times to each observer.

The nonius fixation was presented at the beginning of each experimental trial. The observers fixated the nonius T-shapes, and only after they had obtained a stable view of a cross with an aligned vertical line did they press a key on the keyboard to start the trial. The first stimulus was presented $2,000 \mathrm{msec}$ after the observer's keypress. After the second stimulus was presented at one of the nine SOAs, the observer pressed a key to report whether the nearer or the more distant stimulus had been perceived later. The two stimuli disappeared when the observer pressed the key.

The observers were told that fixation was their primary instruction and that they should try to keep the parts of the fixation cross aligned throughout the trial. That condition was presented again at the end of the block if there were any problems in fixation in a trial. 


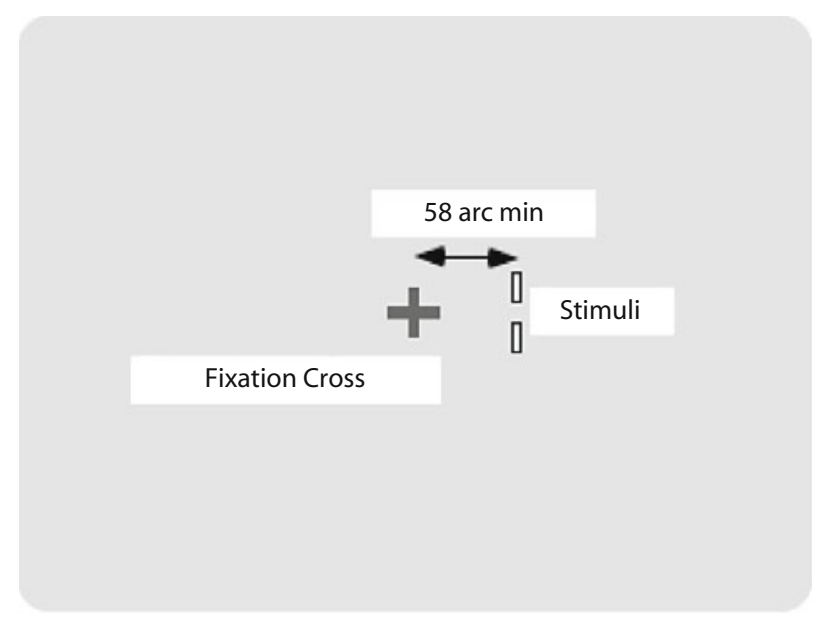

Figure 4. Stimulus configuration used in Experiment 2. Two white stimuli were presented at the right side of a fixation point.

To familiarize themselves with the equipment and procedure, observers had a training session with at least 20 trials before the experimental trials. All sessions, including the training sessions, for an observer lasted about $60 \mathrm{~min}$.

Observers. The author and 4 new naive participants served as observers in the second experiment ( 1 female and 4 male). All 5 observers had normal or corrected-to-normal visual acuity. In a preliminary test using the same stimuli as in Experiment 2, all showed correct depth order perception for the two stimuli in almost all observations (at least $97 \%$ of the observations).

\section{Results and Discussion}

No observer reported any difficulty in fixating and achieving a stable view of the fixation cross and two stimuli during the experimental session, which implies that observers were able to fixate the fixation nonius. There was no consistent effect of depth order condition (upper or lower stimulus nearer the observer), as in Experiment 1. Therefore, the results for this condition were combined in the following analyses.

Figure $5 \mathrm{~A}$ portrays the frequency of the trials in which the observer perceived that the presentation of the nearer stimulus followed the presentation of the more distant stimulus, for 2 observers. To obtain the SOA for the simultaneous perception of the presentations of the two stimuli, a probit analysis was conducted for the individual observers' data for each depth position condition. Figure $5 \mathrm{~B}$ presents the means of the SOAs at which, in $50 \%$ of the trials, the observer perceived the presentation of the nearer stimulus as following the presentation of the more distant stimulus for each condition (a positive value of the SOA indicates that the presentation of the nearer stimulus was earlier than that of the more distant stimulus). In Figure 5B, the means and the $t$ statistic $(p<.05)$ suggest that the SOAs were significantly larger than zero in the around-fixation-plane, in-front-of-fixation-plane, and nearest conditions, although the SOAs in the most distant and behind-fixation-plane conditions were not significantly different from zero. These results closely resemble those of Experiment 1, indicating that the positive and large SOAs for the stimuli presented at depth posi- tions around or in front of the fixation were not caused by inaccurate fixation or mismatch of the stimulus images.

We conducted a one-way repeated measures ANOVA for the condition of depth position using the SOA data from the 5 observers (Figure 5B). The main effect of condition was significant $[F(4,16)=7.438, p<.001]$. Tukey's post hoc tests showed that the SOAs in the behindfixation-plane and most distant conditions were significantly smaller than those in the around-fixation-plane, infront-of-fixation-plane, and nearest conditions $(p<.05)$.

One reviewer pointed out the possibility that, because we used a relatively long duration for stimulus presentation, the observers may have executed vergence eye movements, which would serve to alter the magnitude and possibly the sign of the disparity information, thus rendering any interpretation of the results in terms of crossed versus uncrossed disparity processing ambiguous. Therefore, we duplicated the second experiment, with each stimulus presented for one frame $(16 \mathrm{msec})$, and with the interval between the start key and presentation of the first stimulus shortened to $432 \mathrm{msec}$. The author and 6 naive observers took part in this additional experiment. Figure 5C shows the SOA data. Comparing Figures 5B and 5C, one can see that the short exposure duration exaggerated the observer's tendency to perceive the nearer stimulus as following the more distant stimulus. A one-way repeated measures ANOVA for the condition of depth position found that the main effect of condition was significant $[F(4,24)=3.821$, $p<.05]$. Tukey's post hoc tests showed that the SOAs in the most distant condition were significantly smaller than those in the in-front-of-fixation-plane and nearest conditions $(p<.05)$. These results for the additional condition indicate that the temporal bias was not caused by inaccurate vergence eye movement during the longer stimulus presentation.

\section{EXPERIMENT 3}

Does the delay in the processing for the nearer stimulus mean that the time course of visual processing is always delayed for the space that is nearer to the observer than the fixation point is? Or does this delay depend on the suddenly changing stimulus attributes, rather than on the general characteristics of temporal structure in our visual experience? To answer these questions, in the third experiment we assessed the time lag that was necessary for the perception of simultaneity for various stimulus attributes that changed suddenly at different depth positions.

\section{Method}

Stimuli. We used the same apparatus as in Experiments 1 and 2. Because we found similar relationships between depth position and apparent temporal order for both horizontal (Experiment 1) and vertical (Experiment 2) stimulus alignment, we used the horizontal alignment in the subsequent experiments. The arrangement of the equipment, the configuration and luminance of the stimuli, the background, and the fixation point were as described for Experiment 1.

In three attribute conditions, observers were asked to judge the time order for the change in different stimulus attributes, whereas in an additional condition, observers were asked to judge the direction of motion. In the first attribute condition (the sudden presentation 

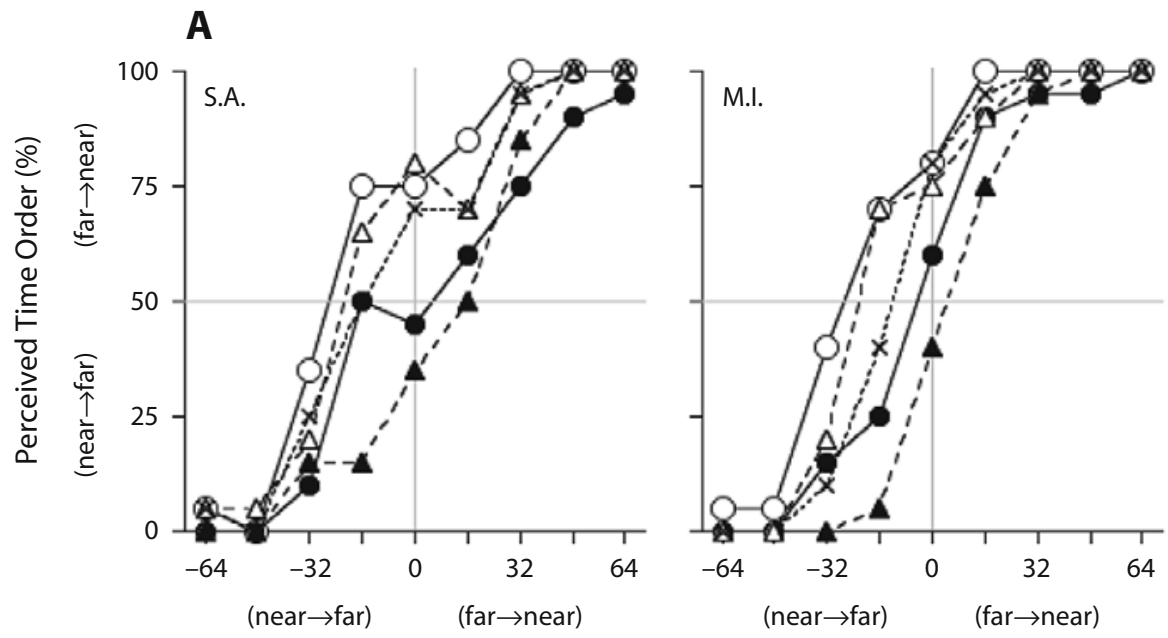

Depth Position Conditions

- Most distant

$\boldsymbol{- 1}-\boldsymbol{B}$ Behind fixation plane

**-.. Around fixation plane

$-\Delta-\ln$ front of fixation plane

$-\mathrm{O}-$ Nearest

$\mathrm{SOA}(\mathrm{msec})$

B

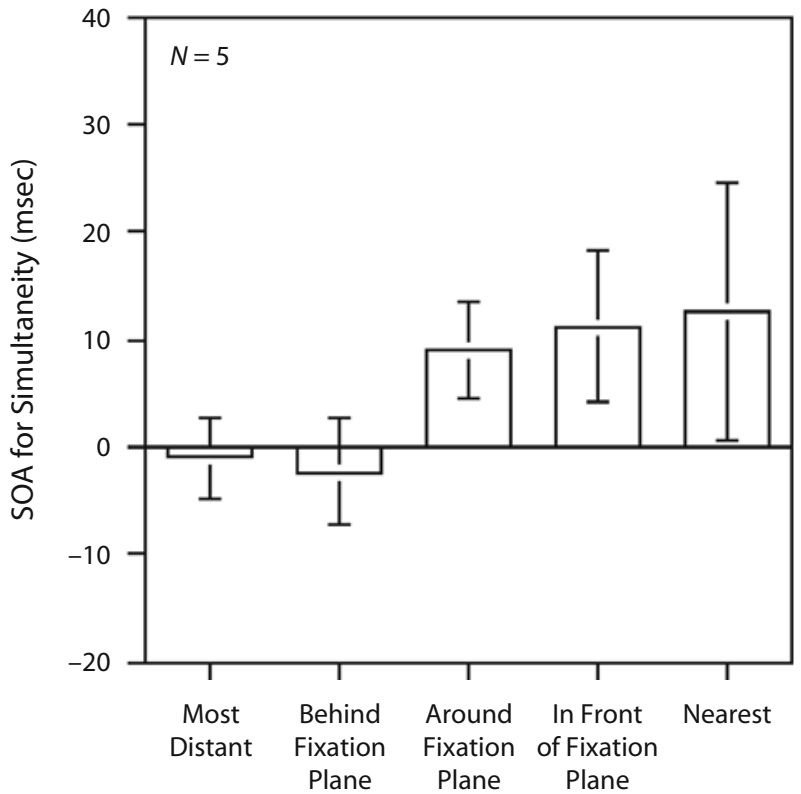

Depth Position Conditions
C

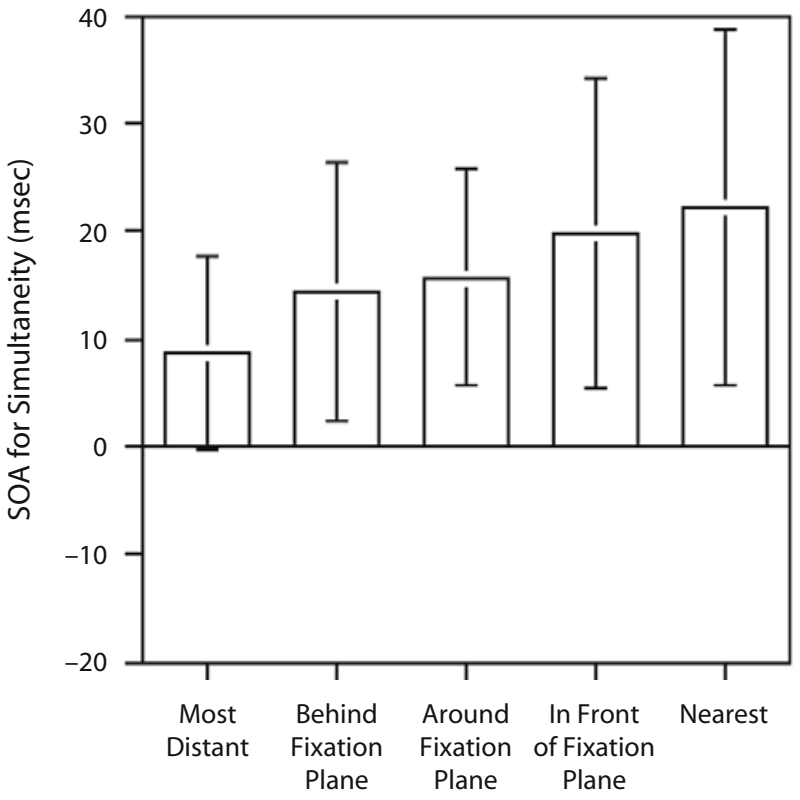

Depth Position Conditions

Figure 5. Results of Experiment 2. (A) Examples for 2 observers (S.A. and M.I.): Frequency with which the presentation of the relatively nearer stimulus was perceived to follow that of the more distant stimulus for each depth position condition. (B) Mean and 95\% confidence limit of the stimulus onset asynchrony (SOA) at which the two stimuli appeared to be presented at the same time in each of the depth position conditions. Positive (or negative) values indicate that the presentation of the relatively nearer stimulus should precede (or follow) the presentation of the more distant one to achieve apparent simultaneity for the presentations of the two stimuli. (C) Results of additional experiment: Mean and $95 \%$ confidence limit of the SOA at which the two stimuli appeared to be presented at the same time in each of the depth position conditions.

condition), two stimuli were presented suddenly at different depth positions with different SOAs, as in Experiment 1 (Figure 6A). In the second attribute condition (the shift position condition), the stimuli were presented at different depth positions from the beginning of the trial (Figure 6B); they shifted independently to the left or right on the front parallel plane. The shift size was 2.9 arc min for both stimuli. In the third attribute condition (the color change condition), two green stimuli were presented at depth positions that were different from those at the beginning of the trial; their color changed independently from green to blue (Figure 6C). The green and blue luminances were equated for each observer. In these second and third attribute conditions, the two stimuli were presented from the beginning of the trial at different depth positions; therefore, no sudden stimulus emergence existed. In the fourth attribute condition (the motion-in-depth condition), each stimulus was presented suddenly for $16 \mathrm{msec}$, with different SOAs at different depth positions (Figure 6D).

Three conditions were used for depth positions of the stimuli (the same conditions as the center three conditions in Figure 1C). In each 
A

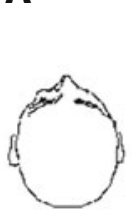

Time 1

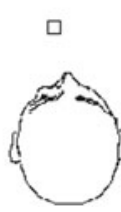

Time 2
ㅁ

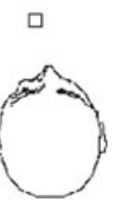

Time 3
B $\square$<smiles></smiles>

Time 1
ㅁ

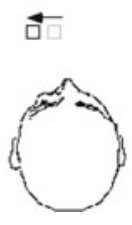

Time 2

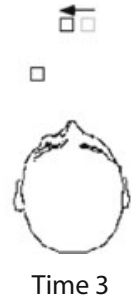

$\square$ Blue $\square$ Blue

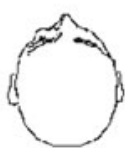

Time 3
D

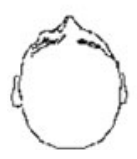

Time 1

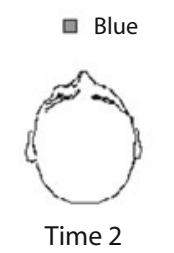

口

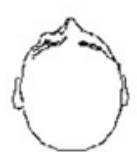

Time 2
ㅁ

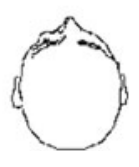

Time 3

Figure 6. Attribute conditions used in Experiment 3. (A) Sudden presentation condition. (B) Shift position condition. (C) Color change condition. (D) Motion-in-depth condition. These diagrams show the case in which the presentation of the relatively more distant stimulus follows the presentation of the relatively nearer stimulus. Observers pressed a key to start the trial (Time 1). One of the stimuli was presented (Time 2), and with a specific SOA, the second stimulus was presented (Time 3).

of the four attribute conditions, the two stimuli were presented at different depth positions, which were specified by 5.8 arc min of binocular disparity, as in Experiments 1 and 2.

Procedure. The sudden presentation condition included 7 asynchrony conditions between the presentations of the nearer and more distant stimuli, ranging from -48 to $+48 \mathrm{msec}$ in $16-\mathrm{msec}$ increments, as in Experiment 1 (a positive value indicates that the presentation of the nearer stimulus followed that of the more distant one). Ten blocks were used for each observer. In a block, 42 stimulus conditions were presented in random order: 7 SOAs $\times 2$ depth orders $\times$ 3 depth positions of the stimuli. The observer's task in each trial was the same as that described for Experiment 1. At the beginning of each trial, the fixation point was presented. The observer pressed a key on the keyboard to start the trial. Following a random interval of 2,000-2,256 msec after the observer had pressed the key, the first stimulus was presented. After the second stimulus was presented

at 1 of the $7 \mathrm{SOAs}$, the observer pressed a key to report which of the stimuli was perceived later. The stimuli disappeared when the observer pressed the key.

The shift position condition included 9 asynchrony conditions between the position shifts at the nearer and more distant stimuli, ranging from -64 to $+64 \mathrm{msec}$ in 16-msec increments (a positive value indicates that the position shift of the nearer stimulus followed that of the more distant one). Ten blocks were used for each observer. In a block, 108 stimulus conditions were presented in random order: 9 asynchronies in the stimulus position shift $\times 2$ depth orders (left or right stimulus nearer to observer) $\times 3$ depth positions of the stimuli $\times 2$ motion directions. At the beginning of each trial, the two stimuli were presented with the fixation point. The observer pressed a key on the keyboard to start the trial. Following a random interval of 2,000-2,256 msec after the observer had pressed the key, the first stimulus's position was shifted to the left or right. After the second stimulus shifted in the same direction as did the first at one of the 9 asynchrony conditions, the observer pressed a key to report which stimulus had shifted later. The stimuli for the next trial were presented when the observer pressed the key.

In the color change condition, initially, 9 asynchrony conditions were used between the color change at the nearer and more distant stimuli, ranging from -64 to $+64 \mathrm{msec}$ in $16-\mathrm{msec}$ increments (a positive value indicates that the color change in the nearer stimulus followed that in the more distant stimulus). All the observers found the task to be difficult when presented with small asynchronies between color changes in the two stimuli. For that reason, we used 9 additional asynchrony conditions ranging from -256 to +256 msec in 64-msec increments. Ten blocks were used for each of two ranges in the asynchrony conditions. In a block, 54 stimulus conditions were presented in random order: 9 asynchronies in the stimulus color change $\times 2$ depth orders $\times 3$ depth positions of the stimuli. At the beginning of each trial, two green stimuli were presented with the fixation point. The observer pressed a key on the keyboard to start the trial. Following a random interval of 2,000$2,256 \mathrm{msec}$, the first stimulus changed its color from green to blue. After the second stimulus changed its color to blue at a specific asynchrony, the observer pressed a key to report which of the stimuli had changed color later. The green stimuli for the subsequent trial were presented when the observer pressed the key.

For the motion-in-depth condition, initially, 9 asynchrony conditions were used between the color change at the nearer and more distant stimuli, ranging from -64 to $+64 \mathrm{msec}$ in 16-msec increments (a positive value indicates that the presentation of the nearer stimulus followed that of the more distant one). All the observers, except for M.I., found the task to be difficult with short asynchronies between the presentations of the two stimuli. For that reason, 9 additional asynchrony conditions were used, ranging from -256 to $+256 \mathrm{msec}$ in 64-msec increments. Ten blocks were used for each of two ranges in the asynchrony conditions. In a block, 54 stimulus conditions were presented in random order: 9 asynchronies in the stimulus presentation $\times 2$ depth orders $\times 3$ depth positions of the stimuli. At the beginning of each trial, the fixation point was presented. The observer pressed a key to start the trial. Following a random interval of 2,000-2,256 msec after the observer had pressed the key, the first stimulus was presented for $16 \mathrm{msec}$. After the second stimulus was presented for $16 \mathrm{msec}$ at one of the 9 asynchronies, the observer pressed a key to report the direction of the apparent motion in depth (approaching or retreating).

To familiarize themselves with the equipment and tasks, observers had training sessions with at least 20 trials before the experimental trials for each attribute condition. Including training, sessions for an observer lasted about $240 \mathrm{~min}$. They were conducted on separate days.

Observers. The author, 2 naive participants (female) from the first experiment, and 2 new naive participants (female) took part in the experiment. All 5 observers had normal or corrected-to-normal visual acuity. In a preliminary test that used the same stimuli as 


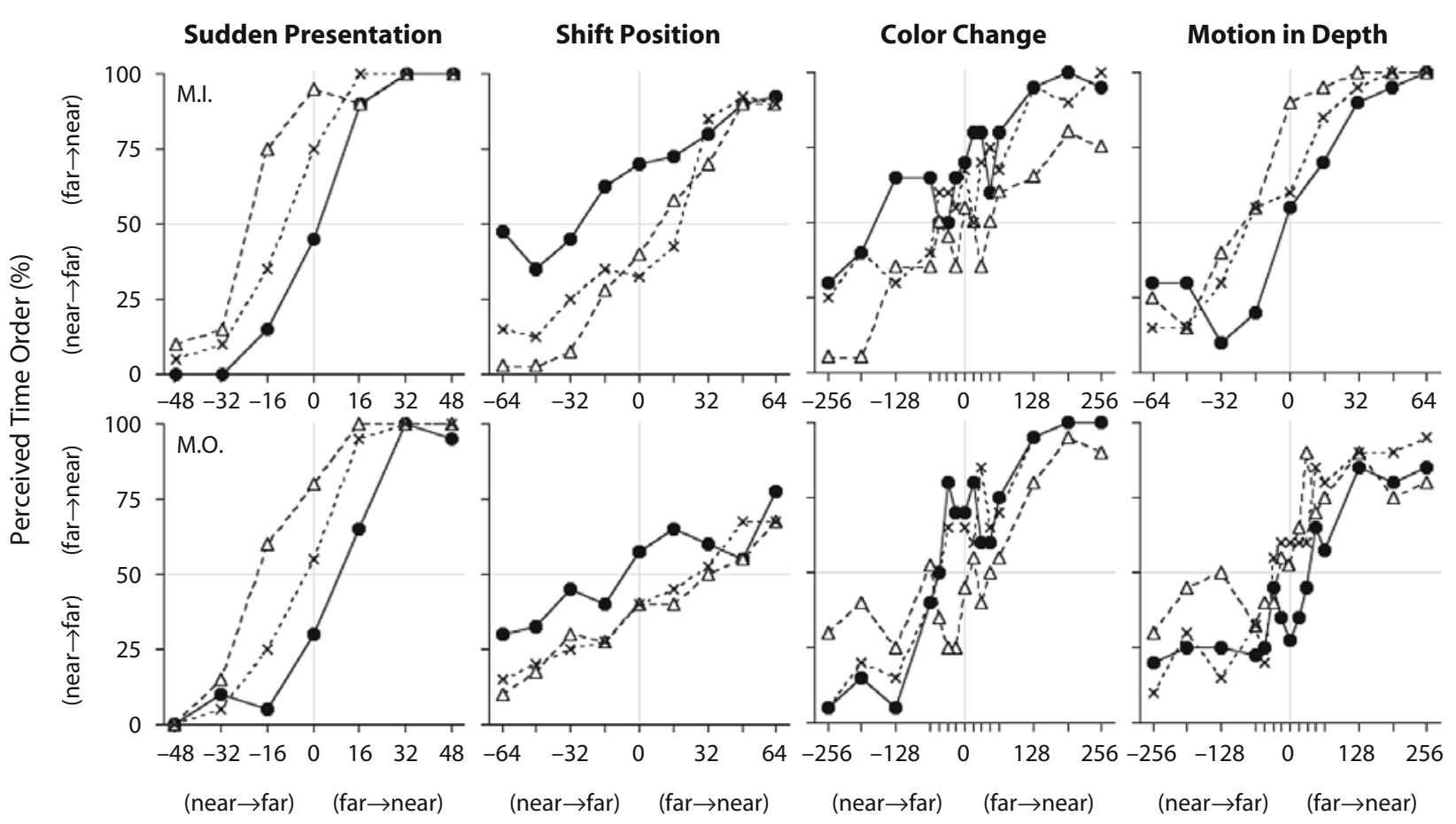

Asynchrony for the Relatively Nearer and Farther Stimuli (msec)

Figure 7A. Results of Experiment 3: Example for 2 observers, M.I. (upper) and M.O. (lower). Closed circle, cross, and open triangle show the results of the behind-, around-, and in-front-of-fixation-plane conditions, respectively.

those in the sudden presentation condition, the 2 new participants showed correct depth order perception for the two stimuli in at least $95 \%$ of the trials.

\section{Results and Discussion}

We did not find a consistent effect of depth order condition (left or right stimulus nearer the observer) on the apparent temporal order in any attribute condition. In addition, the shift position condition revealed no consistent effect of direction of the position shift. Therefore, the results of these conditions were combined in the following analyses.

Figure 7A portrays the frequency with which the change for the nearer stimulus was perceived to follow the change for the other stimulus in each attribute condition, for $2 \mathrm{ob}-$ servers. A probit analysis of the respective observer's data determined the asynchrony at which the observers perceived that the changes in the nearer and more distant stimuli were simultaneous. Figure $7 \mathrm{~B}$ shows the means of the time lags in each condition for 5 observers. As is shown in Figure 7B, the means and the $t$ statistic $(p<.05)$ suggest that the asynchronies between the two changes at different depth positions were significantly larger than zero in the sudden presentation and motion-in-depth conditions. The large $95 \%$ confidence limits in the shift position and color change conditions indicate no consistent tendency in the asynchrony among observers.

A one-way repeated measures ANOVA was performed for the asynchrony of the changes at different depth positions for each attribute condition, with depth position as a factor. A significant main effect of depth position condition was found for the sudden presentation $[F(2,8)=$ 9.457, $p<.01]$ and for the motion-in-depth condition $[F(2,8)=9.612, p<.01]$, although no significant effect of depth position was found in the other two conditions. For both the sudden presentation and motionin-depth conditions, Tukey's post hoc $t$ tests revealed significant differences between the most distant and the nearest depth position conditions $(p<.01)$. These results indicate that time in visual space was not delayed consistently for all stimulus attributes in the space nearer the observer. Rather, the consistent delay for the stimuli presented at the nearer depth position depended on the stimulus attributes.

In the motion-in-depth condition, for which they were asked to report the perceived direction of motion, observers tended to perceive an approaching motion when the two stimuli were presented simultaneously at different depth positions (Figure 7B). That perception indicates that the processing for motion in depth, which may be triggered by a sudden change of luminance in stimuli, produces a bias to perceive motion toward the observer when suddenly presented stimuli are ambiguous in defining the direction of motion. This bias in the processing for motion in depth is expected to be similar to the illusory temporal bias found in Experiment 1 for the presentations of two stimuli at different depth positions. These biases might share a common basis involved in the processing for judgment of simultaneity when a sudden change of luminance occurs in two stimuli. 
Sudden Presentation

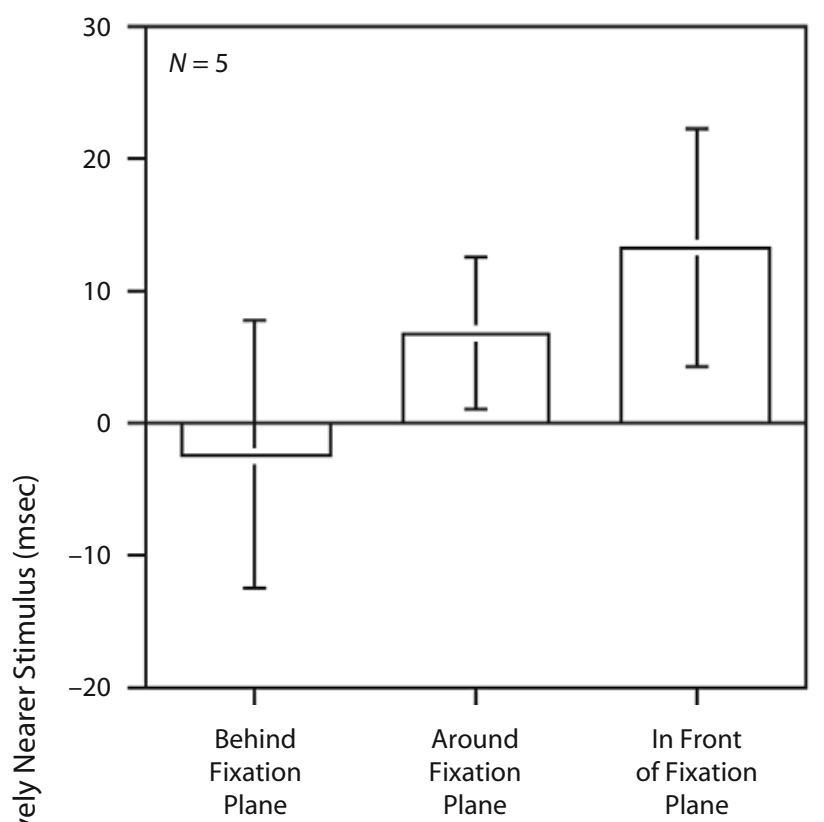

Color Change

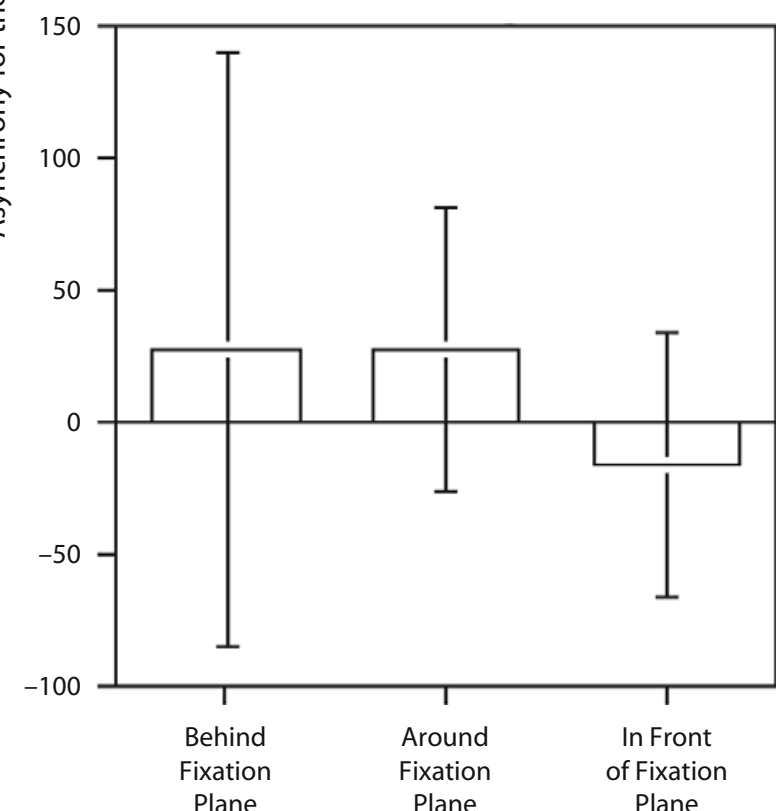

Depth Position Conditions
Shift Position

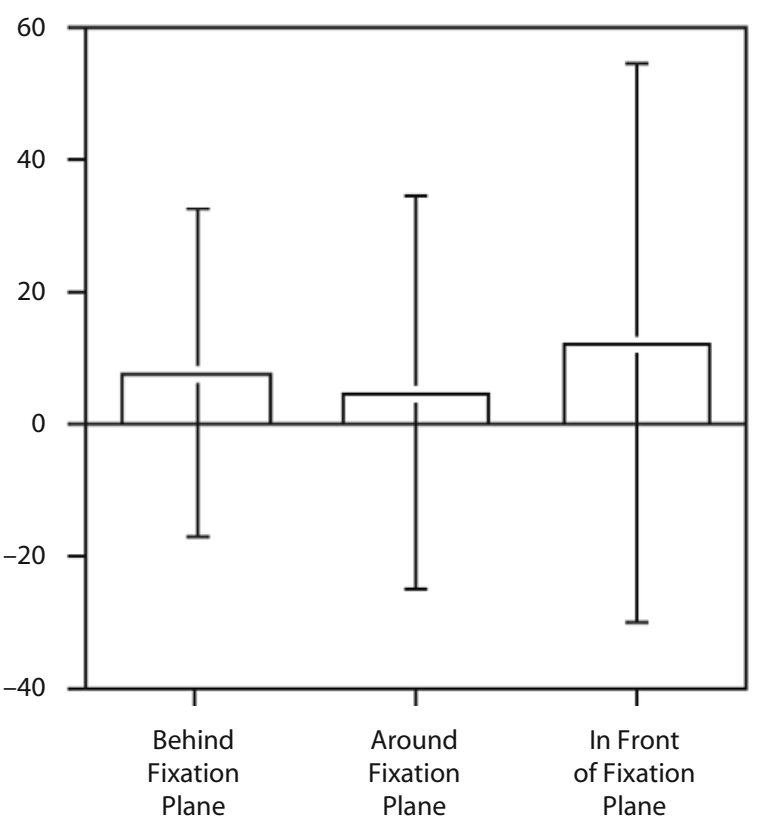

Motion in Depth

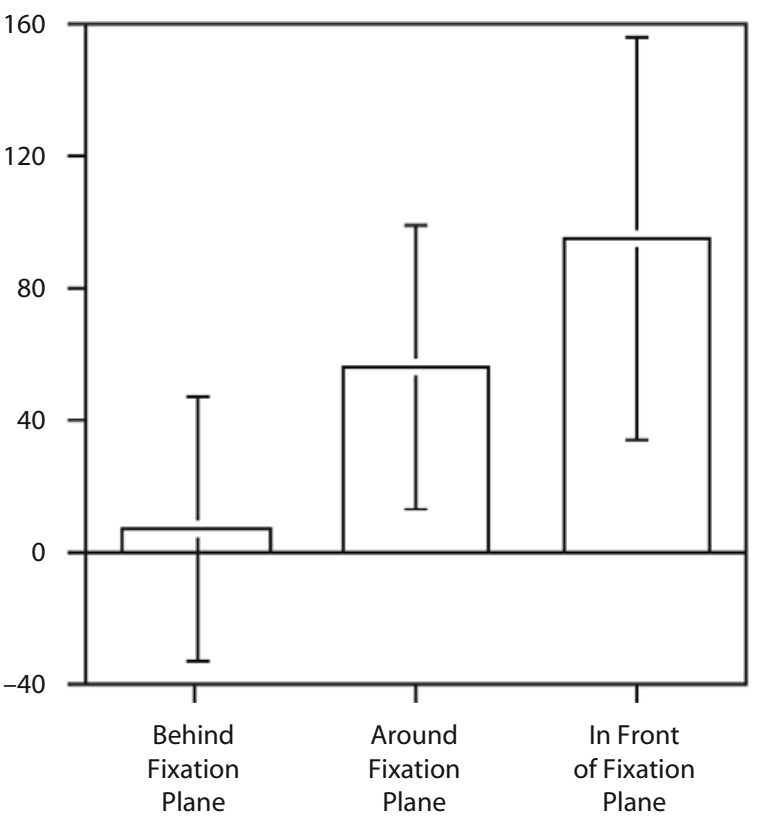

Depth Position Conditions

Figure 7B. Results of Experiment 3: Mean and $95 \%$ confidence limit of the time lag at which the changes appeared to happen at the same time for the two depth positions in each attribute conditions. Positive (or negative) values indicate that the presentation of or change in the relatively nearer stimulus should precede (or follow) that for the more distant one to achieve apparent simultaneity for the presentations of or the changes in the two stimuli.

\section{EXPERIMENT 4}

In Experiment 1, the extent of the illusory temporal bias increased with the increment in the distance between the stimuli and fixation plane. This result suggests that the il- lusory temporal bias depends on the depth position of the suddenly presented stimuli. The positions of the stimuli in the depth dimension are defined by the disparity size. Therefore, it is likely that the illusory temporal bias is affected by the disparity size of the stimulus. Burt and Julesz 
(1980) demonstrated that binocular fusion in stereopsis depends on the disparity gradient. That is, if one views two stimuli having a disparity gradient greater than 1 , the images of only one stimulus can be fused. More important for the present study, the disparity gradient specifies the direction of the trajectory, which goes through the stimuli. A large gradient, such as 2, implies that the trajectory should go through the observer's head, whereas a small gradient implies that the trajectory will turn away from the observer's head. The results of Experiment 3 suggested that the processing that underlies the directional bias in the processing of motion in depth may be involved in the illusory delay for the nearer stimulus. If an object is approaching an observer, the disparity gradient, which specifies the trajectory, will determine the possibility of collision with the object. Therefore, it is plausible that the disparity gradient affects the illusory temporal bias. Experiment 4 examined the degree to which the illusory temporal bias depends on disparity size and the disparity gradient.

\section{Method}

Stimuli and Apparatus. We used the same apparatus as in Experiment 1 . The configuration and luminance of the stimuli, background, and fixation point were identical to those used in Experiment 1.

There were three disparity size/gradient conditions defined according to disparity size and disparity gradient. For each of the three conditions, we prepared five conditions for the depth position of the stimuli, defined by the depth position of the middle point between the two stimuli: most distant (corresponding to uncrossed 11.6 arc min of disparity), behind fixation plane (corresponding to uncrossed 5.8 arc min of disparity), around fixation plane (corresponding to 0.0 arc min of disparity), in front of fixation plane (corresponding to crossed 5.8 arc min of disparity), and nearest (corresponding to crossed 11.6 arc min of disparity).

The first disparity size/gradient condition was the basic condition. In this condition, the disparity size between the two stimuli was 5.8 arc min; the disparity gradient was $2 / 3$. The stimuli were the same as those used in the five depth position conditions in the preceding three experiments (Figure 1C). The second condition was the small-disparity-gradient condition. In this condition, although the disparity size between the two stimuli was 5.8 arc min, the dispar- ity gradient was reduced to $2 / 15$. The stimuli were presented on the same depth planes as those in the basic condition (those planes are shown in Figure 1C) in all the depth position conditions, whereas the horizontal distance between the stimuli was five times the horizontal distance in the basic condition. The third condition was the largedisparity condition. In this condition, the disparity size between the two stimuli was magnified to 11.6 arc min, although the disparity gradient was fixed at $2 / 3$. The middle points between the two stimuli in the five depth position conditions were presented on the same depth planes as those in the other two conditions (Figure 8).

Procedure. Nine SOA conditions were used for the presentation of the two stimuli, ranging from -64 to $+64 \mathrm{msec}$ in 16 -msec increments (a positive value of the SOA indicates that the presentation of the nearer stimulus preceded that of the more distant stimulus). In all, 30 blocks were used for each observer. In a block, the disparity size/gradient condition was constant. The disparity size/gradient condition for each block was determined in random order. In a block, 90 stimulus conditions were presented in random order: 9 presentation SOAs $\times 2$ depth orders $\times 5$ depth positions of the stimuli.

The fixation point was presented at the beginning of each trial. The observer pressed a key on the keyboard to start the trial. Following a random interval of 2,000-2,256 msec after the observer had pressed the key, the first stimulus was presented. After the two stimuli were presented at a specific SOA, the observer pressed a key to report which of the stimuli had been perceived later. The display returned to the initial stage that presented only the fixation point when the observer pressed the key. To familiarize themselves with the equipment and tasks, observers had training sessions with at least 20 trials before the experimental trials. Sessions for an observer, including the training sessions, lasted about $180 \mathrm{~min}$. They were conducted on separate days.

Observers. The author and a naive participant (female) from Experiments 1 and 3 , along with 3 new naive participants ( 1 female and 2 male) took part in the experiment. All observers had normal or corrected-to-normal visual acuity. The results of a preliminary test, which used the same stimuli as those used in the basic condition, revealed that the 3 new observers had correct depth order perception for the stimuli in at least $96 \%$ of the trials.

\section{Results and Discussion}

We did not find a consistent effect of depth order in any condition. Therefore, the results of this condition were combined in the following analyses.

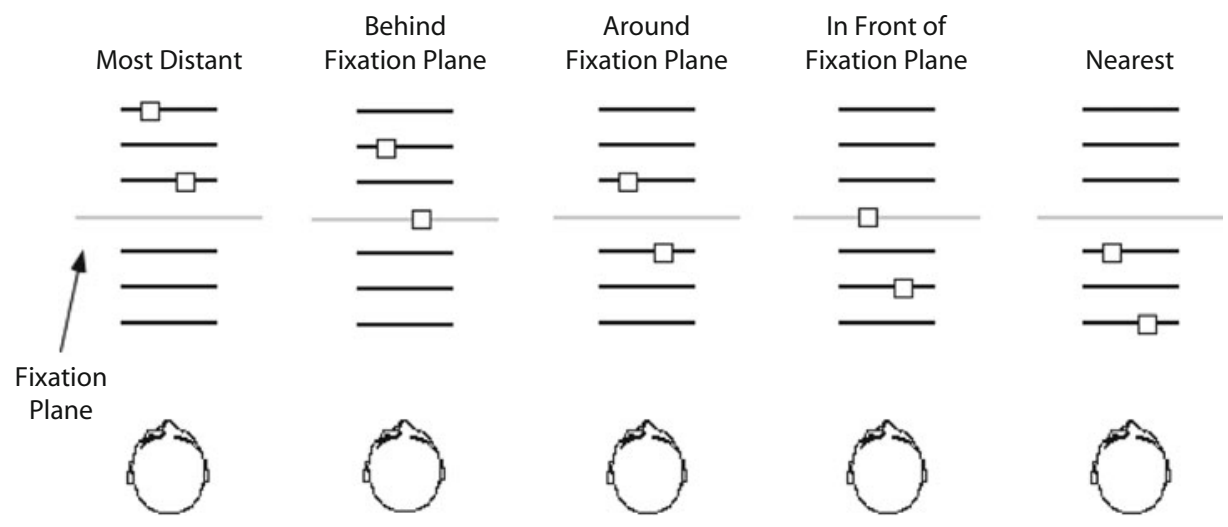

Figure 8. Five depth position conditions for the large-disparity-size condition in Experiment 4. There were seven depth planes defined by binocular disparity relative to the fixation plane (uncrossed, 17.4 arc min; uncrossed, 11.6 arc min; uncrossed, 5.8 arc min; 0.0 arc min; crossed, 5.8 arc min; crossed, 11.6 arc min; and crossed, 17.4 arc min). The two stimuli were presented on the two depth planes so that the disparity between the stimuli was 11.6 arc min. This diagram shows the case in which the right stimulus is relatively nearer than the other stimulus. 
A

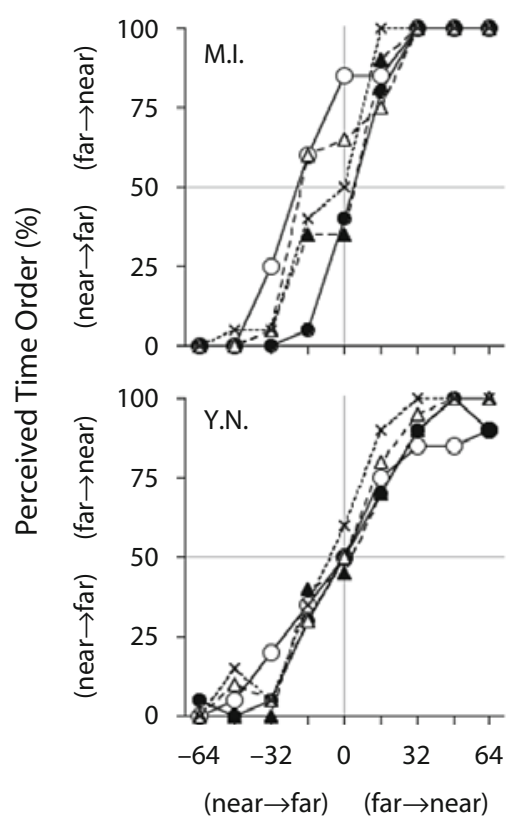

Small Disparity Gradient
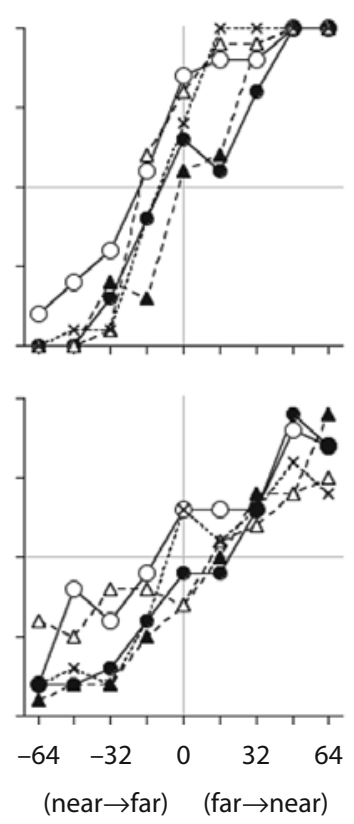

SOA (msec)

\section{Large Disparity Size}

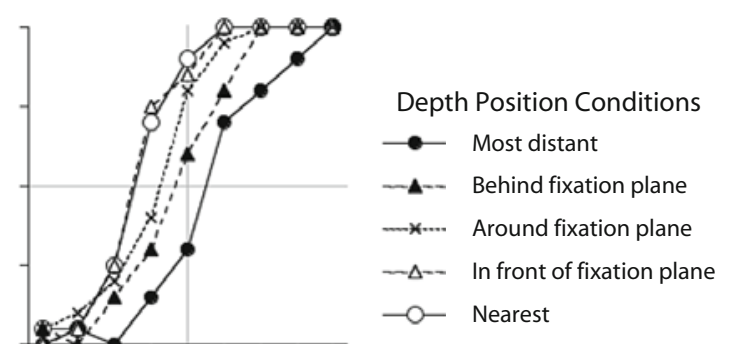

B

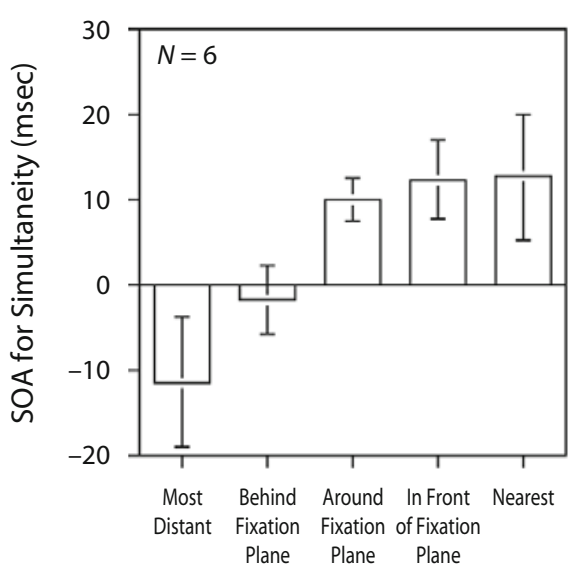

Small Disparity Gradient

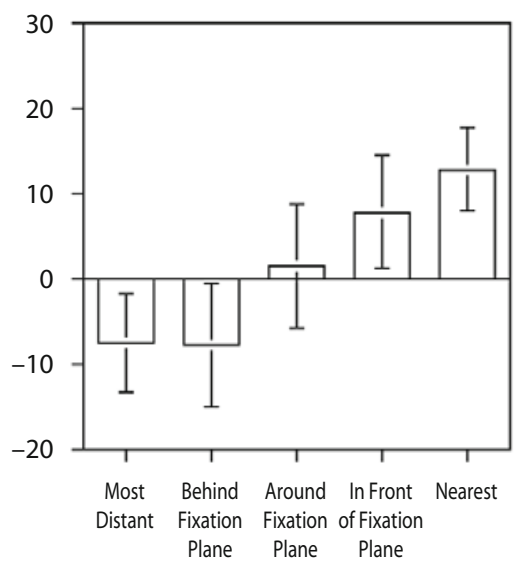

Depth Position Conditions
Large Disparity Size

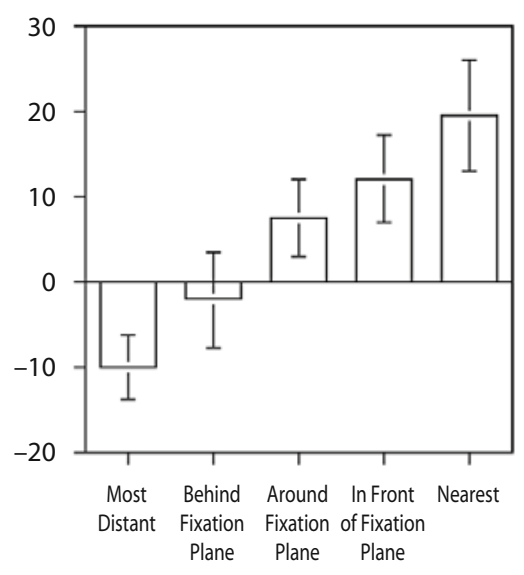

Figure 9. Results of Experiment 4. (A) Example for 2 observers: M.I. (upper) and Y.N. (lower). (B) Mean and 95\% confidence limit of the stimulus onset asynchrony (SOA) for the perception of simultaneity in each condition. Positive (or negative) values indicate that the presentation of the relatively nearer stimulus should precede (or follow) the presentation of the more distant one to achieve apparent simultaneity for the presentations of the two stimuli.

Figure 9A shows the frequency of the trials in which the observer perceived that the presentation of the nearer stimulus followed the presentation of the more distant stimulus, for Observers M.I. (upper row) and Y.N. (lower row). A probit analysis determined the SOA at which the observer perceived that the respective presentations of the stimuli were simultaneous. Figure 9B portrays the mean and $95 \%$ confidence limit of the SOA from 6 observers for each of three disparity size/gradient conditions. In Figure 9B, the means and the $t$ statistic $(p<.05)$ show that the SOAs were significantly larger than zero in the aroundfixation-plane condition for the basic and large-disparity conditions, although the SOAs were not significantly different from zero in the around-fixation-plane condition for the small-disparity-gradient condition. These results indicate that the illusory temporal biases around the fixation plane, which were found in the other experiments, would disappear for the small disparity gradient.

A two-way repeated measures ANOVA was conducted, with disparity size/gradient condition and depth position 
condition for the two stimuli as factors for the SOA for visual simultaneity. The main effect of depth position was significant $[F(4,12)=55.490, p<.001]$, although the main effect of disparity size/gradient was not significant $[F(2,6)=4.051, p>.05]$. There was a significant interaction of the two factors $[F(8,24)=3.138, p<.05]$. Tukey's post hoc HSD test for the simple main effect of disparity size/gradient condition showed a significant difference between the basic and large-disparity conditions in the nearest condition, and between the basic and smalldisparity-gradient conditions in the around-fixation-plane condition $(p<.05)$. These results indicate that a large disparity size exaggerated the illusory temporal bias for the space nearer than the fixation plane and that a small disparity gradient reduced the illusion around the fixation point. No other significant difference attributed to the disparity size/gradient conditions was found at any depth position.

\section{GENERAL DISCUSSION}

The results of the present Experiment 1 add the depth position specified by a binocular disparity cue to the list of factors that affect visual simultaneity. That is, when two stimuli were presented abruptly at different depth positions, the observer tended to perceive that the stimulus more distant from the fixation point appeared after the other stimulus (Figure 3B). The means and 95\% confidence limits in Figure 3B, as well as those in Figures 5B and $9 \mathrm{~B}$, show that this tendency was unclear when the stimuli were more distant from the fixation point. In addition, those figures showed that the observer perceived that the stimulus nearer to the observer appeared after the more distant stimulus in the around-fixation-plane condition. These results indicate that, although observers tended to perceive that the stimulus more distant from the fixation point appeared after the other stimulus, they had an inclination to perceive that the nearer stimulus appeared after the more distant one. The results for the additional condition in Experiment 2 (Figure 5C) show that this inclination was exaggerated when the stimuli were presented for a very short duration $(16 \mathrm{msec})$.

The tendency of the observers to perceive the nearer stimulus after the other stimulus apparently contradicts results from studies that have shown temporal biases in visual perception because of the depth position for a single stimulus with a fixation point. That is, previous studies (Bradshaw et al., 1995; Downing \& Pinker, 1985; Gawryszewski et al., 1987; Howard \& Rogers, 1995, 2002; Landers \& Cormack, 1997; Miura et al., 2002; Mustillo, 1985; Patterson et al., 1995) have shown that processing for a stimulus presented in the space nearer than the fixation plane is faster than processing for a stimulus in the space that is more distant than the fixation plane. The results of Experiment 2, in which observers fixated a nonius, showed larger biases for the stimuli presented around or in front of the fixated depth plane than for the stimuli presented as more distant than the fixation plane, as in Experiment 1 (Figure 5B and 5C). These results indicate that the illusory temporal bias found in Experiment 1 cannot be explained in terms of inaccurate fixation, such as underconvergence.
Is the bias derived from the differences in latency of stimuli at different depth positions? In the large-disparity condition in Experiment 4, the disparity between the stimuli was 11.6 arc min, although it was only 5.9 arc min in the basic condition. Therefore, if the relationship between the disparity size and delay for each stimulus was fixed - that is, if the bias could be derived from the time difference in processing the stimuli - we would be able to estimate approximately the delay for some depth position conditions in the large-disparity condition (right panel in Figure 9B) by summing the SOAs obtained from the two neighboring depth position conditions in the basic condition (left panel in Figure 9B). The SOAs obtained in the large-disparity condition, however, were much smaller than the summation of the SOAs obtained from the two neighboring depth position conditions in the basic condition. For instance, when the SOAs obtained from 6 observers for the middle three depth position conditions in the large-disparity condition were fitted to the SOAs predicted from the results of the basic condition, ${ }^{1}$ the regression was significant $(r=$ $.725, p<.001, N=18$ ), but the regression coefficient (.439) was much smaller than 1 . These results indicate that the illusory temporal bias that we found for the two stimuli in this study cannot be explained by using the summation of the delay for each single stimulus presented in 3-D space and that the bias cannot be derived from the latency for each stimulus.

The empirical horopter, within which an object appears to be located on the same depth plane as is the fixation point, is inclined backward (see, e.g., Grove, Kaneko, \& Ono, 2001; Nakamizo, Ono, \& Ujike, 1999; Nakayama, 1977). Previous studies reported that the angle of the topaway inclination for the vertical horopter was approximately $30^{\circ}$ at a viewing distance of $65 \mathrm{~cm}$ (Grove et al., 2001 ) and $40^{\circ}$ at a viewing distance of $120 \mathrm{~cm}$ (Nakamizo et al., 1999). By substituting these angles into the equation, which describes the relationship between viewing distance and angle of the horopter inclination (Howard \& Rogers, 2002, p. 227), we obtain an inclination angle of, at most, $22^{\circ}$ at a viewing distance of $45 \mathrm{~cm}$, which was used in this study. The stimuli were presented in the upper visual field in Experiments 1, 3, and 4. Therefore, in these experiments, on the one hand, the distance from the horopter to the nearer stimulus would be larger than the distance from the horopter to the more distant stimulus for the stimuli in the nearest, in-front-of-fixation-plane, and around-fixation-plane conditions. On the other hand, the distance from the horopter to the nearer stimulus would be smaller than the distance from the horopter to the more distant stimulus for the stimuli in the behind-fixation-plane and most distant conditions. Considering these facts, we should examine whether the distance between the horopter and each stimulus, rather than the depth positions of the stimuli, determines the apparent temporal order for the stimulus presentations at different depth positions.

Although the distances from the horopter to each stimulus in Experiment 1 were fixed at each depth position condition, because the stimuli were aligned horizontally above the fixation points, the distances from the horopter to each stimulus in Experiment 2 varied with the depth 
order condition (which of the upper or lower stimulus was nearer to the observer), because the stimuli were aligned vertically at the right side of the fixation point (Figure 4). That is, in Experiment 2, the two stimuli were on the line that was inclined backward (when the lower stimulus was nearer to the observer than the upper stimulus) or forward (when the upper stimulus was nearer to the observer) by about $45^{\circ}$. This indicates that the distance from the horopter to each stimulus varied with the depth position and depth order conditions. However, we could find no significant effect of depth order condition on the apparent temporal order, although there was a significant effect of depth position condition in Experiment 2. (For instance, a two-way repeated measures ANOVA with the depth order and depth position conditions as factors for the SOA for visual simultaneity in the additional experiment with short stimulus durations indicated that the main effect of depth order $[F(1,6)=0.548, p=.487]$ and the interaction of the two factors $[F(4,24)=1.4476, p=.249]$ were not significant, although the main effect of depth position was significant $[F(4,24)=2.854, p=.046]$.) The results of Experiment 2 (shown in Figure 5B) were similar to those of Experiment 1 (shown in Figure 3B), although there were big differences in the distance from horopter to stimuli in each depth position condition between these experiments. Moreover, in a preliminary test with 4 observers in which we used methods similar to those used in Experiment 1, except that the stimuli were presented 56.5 arc min below a fixation point in half of the trials, we found that the SOA at which the two stimuli appeared simultaneously varied with the depth position conditions, as in Experiment 1 (the means of the SOA were $61.3 \mathrm{msec}$ [nearest], $25.2 \mathrm{msec}$ [in front of fixation plane], $12.1 \mathrm{msec}$ [around fixation plane], $5.8 \mathrm{msec}$ [behind fixation plane], and $6.2 \mathrm{msec}$ [most distant]), although the relationship in the placement of each stimulus to the horopter was opposite to that in Experiment 1. In accordance with that, we concluded that this difference in placement does not cause the illusory bias for the temporal order of the stimulus presentations.

We may consider the basis of the illusory temporal bias in accordance with the results of Experiments 3 and 4. The results of Experiment 3 indicate that the illusory temporal delay of the nearer stimulus was found only with a sudden presentation of two stimuli. This result implies that the illusory delay does not depend on a general distortion of the spatiotemporal structure of 3-D space in our visual experience; instead, the illusion is stimulus attribute dependent. The bias of the delay for the nearer stimulus was most pronounced for the motion-in-depth condition in Experiment 3. In the additional condition of Experiment 2, in which each stimulus was presented for a very short duration $(16 \mathrm{msec})$, the more distant stimulus appeared before the nearer stimulus in all depth position conditions (Figure $5 \mathrm{C}$ ). These results suggest that a common processing, which is triggered by abrupt luminance change at different depth positions, underlies both the illusory delay for the nearer stimulus and the directional bias in the processing of motion in depth. This notion is compatible with the results of previous studies that have found a preference for approaching motion versus receding motion in visual processing (see, e.g., Ball \& Sekuler, 1980; Lewis \& McBeath, 2004; Shirai \& Yamaguchi, 2004; Takeuchi, 1997) and in attracting attention (Franconeri \& Simons, 2003). A short duration exaggerates this tendency in the processing for motion in depth; consequently, the illusory delay for the nearer stimulus in the additional condition of Experiment 2 was exaggerated. The preference for approaching versus receding motion might reflect a general tendency of the visual system, irrespective of the cue that specifies the motion in depth. That is, visual processing detects the approaching motion signal from the stimuli with small SOAs, for which visual processing might have difficulty detecting the temporal order of their emergence.

The results of Experiment 4 indicate that the extent of the illusory delay of the relatively nearer stimulus in the around-fixation-plane condition depends both on the gradient and on the size of the disparity. The illusory temporal delay of the nearer stimulus in the around-fixation-plane condition was found only for the conditions with the large disparity gradient. For the small-disparity-gradient condition, the apparent delay of the stimulus depended on the distance between the stimulus and the fixation plane. The apparent delay of the stimulus that was more distant from the fixation point would be caused by a decrement of the effect of visual attention. That is, because the observer's fixation point was on the display surface, the facilitation of visual processing as an effect of attention, which was focused on that point, would decrease with the distance in depth dimension from the fixation point to the stimulus. However, the effect of attention cannot explain the illusory temporal delay of the relatively nearer stimulus for the large disparity gradient.

From these results, we presume that the temporal bias depends on the disparity gradient, which is compatible with the finding that the visual system has an acute ability to distinguish objects headed directly toward an observer from those that will miss the observer (see, e.g., PortforsYeomans \& Regan, 1996). In the small-disparity-gradient condition, the specified motion trajectory was turned away from the center of the observer's face by about $45 \mathrm{~cm}$; however, in the basic and large-disparity conditions, the motion trajectory went through a point about $3 \mathrm{~cm}$ to one side of the center of the observer's face. Tse and Logothetis (2002) found that high-level motion processing, which analyzes the 3-D shape and trajectory of motion in depth, needs about $100 \mathrm{msec}$ to generate the apparent motion with consistent direction. In Experiment 4, as well as in the other experiments in this study, the SOA for the two stimuli was at most $64 \mathrm{msec}$. Therefore, the results of Experiment 4 suggest that the temporal bias in the large disparity gradient would be in the nature of low-level processing of motion, such as motion signal detection, rather than of high-level processing of motion, which analyzes the shape and trajectory of a motion stimulus (see Tse, Cavanagh, \& Nakayama, 1998).

The small temporal bias in the small-disparity-gradient condition and the large bias in the large-disparity condition imply that the extent of the illusory temporal bias might be related to the probability of a collision with a suddenly emergent object, which would be assessed in 
the early stage of visual processing. An interpretation of the illusory temporal bias in terms of the fast processing of the direction of motion in depth posed by a suddenly presented stimuli would be helpful in solving the apparent disagreement between the results of this study, which used two stimuli, and the results of previous studies, which used a single stimulus (see, e.g., Bradshaw et al., 1995; Downing \& Pinker, 1985; Gawryszewski et al., 1987; Howard \& Rogers, 1995, 2002; Miura et al., 2002; Mustillo, 1985; Patterson et al., 1995). All of these biases in terms of the depth order found in the present and previous studies indicate that the visual system is equipped to prepare for the sudden and probable collision with a stimulus in the space in front of the fixation plane.

The introduction of this article includes a list of factors that affect the temporal relationship in visual perception. The results of the present experiments add the depth position specified by a binocular disparity cue to this list. Because, in this study, the fixation point was always at the center of the display surface, the apparent depth position of each stimulus was not separated from the specific disparity size. Future study should examine how each of the apparent depth positions and the disparity size, as a single factor, affect the apparent temporal order of presentations of two stimuli at different depth positions.

We must note the importance of the factor of depth position, in comparison with those other factors, in understanding the spatiotemporal nature of our visual experience. Two different stimuli cannot occupy the same spatial position in physical space simultaneously. The apparent temporal order for stimuli that are suddenly presented separately from each other on a front parallel plane is known to vary according to the retinal positions of the stimuli (see, e.g., Ichikawa, 2002; Mitrani et al., 1986). It is also known that processing speed varies with eccentricity even if the cortical representation sizes of the stimuli are equal (see, e.g., Carrasco, McElree, Denisova, \& Giordano, 2003). Regarding visual processing, the mapping of stimuli from the physical spatiotemporal coordinate to the perceptual spatiotemporal coordinate systematically modulates the temporal relationship between stimuli according to the spatial position of the stimuli. This modification indicates that the physical relationship in the temporal dimension for stimuli presented at different 3-D positions cannot be preserved during mapping. The perceived temporal relationships among stimuli that emerge suddenly at mutually separated positions in visual space are expected to always be different from their physically defined temporal relationships, because the perceived temporal relationship varies with the spatial positions of the stimuli.

\section{AUTHOR NOTE}

This research was partially supported by the Japanese Society for the Promotion of Science. Parts of the data were presented at the annual meeting of the Vision Sciences Society, Sarasota, FL, May 2002 and May 2003, and at the Asian Conference on Vision, Chongqing, China, November 2004. The author thanks R. Peterson, J. Wolfe, and an anonymous reviewer for their helpful comments and suggestions on earlier versions of the manuscript. Address correspondence to M. Ichikawa, Department of Psychology, Chiba University, 1-33 Yayoi, Inage, Chiba, 263-2283, Japan (e-mail: ichikawa@1.chiba-u.ac.jp).

\section{REFERENCES}

Alvarez, T. L., Semmlow, J. L., \& Pedrono, C. (2005). Divergence eye movements are dependent on initial stimulus position. Vision Research, 45, 1847-1855.

Anstis, S. [M.] (2001). Footsteps and inchworms: Illusions show that contrast affects apparent speed. Perception, 30, 785-794.

Arnold, D. H., Clifford, C. W. G., \& Wenderoth, P. (2001). Asynchronous processing in vision: Color leads motion. Current Biology, 11, 596-600.

Aymoz, C., \& Viviani, P. (2004). Perceptual asynchronies for biological and non-biological visual events. Vision Research, 44, 1547-1563.

Bachmann, T., Luiga, I., Põder, E., \& Kalev, K. (2003). Perceptual acceleration of objects in stream: Evidence from flash-lag displays. Consciousness \& Cognition, 12, 279-297.

Baldo, M. V. C., Kihara, A. H., Namba, J., \& Klein, S. A. (2002). Evidence for an attentional component of the perceptual misalignment between moving and flashing stimuli. Perception, 31, 17-30.

BALl, K., \& SEKULER, R. (1980). Human vision favors centrifugal motion. Perception, 9, 317-325

Becker, S., Bowd, C., Shorter, S., King, K., \& Patterson, R. (1999). Occlusion contributes to temporal processing differences between crossed and uncrossed stereopsis in random-dot displays. Vision Research, 39, 331-339.

Bedell, H. E., Chung, S. T. L., Ogmen, H., \& Patel, S. S. (2003). Color and motion: Which is the tortoise and which is the hare? Vision Research, 43, 2403-2412.

Bradshaw, M. F., Rogers, B. J., \& De Bruyn, B. (1995). Perceptual latency and complex random-dot stereograms. Perception, 24, 749-759.

Brenner, E., \& Smeets, J. B. J. (2000). Motion extrapolation is not responsible for the flash-lag effect. Vision Research, 40, 1645-1648.

BurT, P., \& Julesz, B. (1980). Modifications of the classical notion of Panum's fusional area. Perception, 9, 671-682.

Carrasco, M., McElree, B., Denisova, K., \& Giordano, A. M. (2003). Speed of visual processing increases with eccentricity. Nature Neuroscience, 6, 699-700

Downing, C. J., \& Pinker, S. (1985). The spatial structure of visual attention. In M. I. Posner \& O. S. M. Marin (Eds.), Attention and performance XI (pp. 171-187). Hillsdale, NJ: Erlbaum.

Eagleman, D. M., \& SeJnowski, T. J. (2000). Motion integration and postdiction in visual awareness. Science, 287, 2036-2038.

Finney, D. J. (1971). Probit analysis (3rd ed.). Cambridge: Cambridge University Press.

Franconeri, S. L., \& Simons, D. J. (2003). Moving and looming stimuli capture attention. Perception \& Psychophysics, 65, 999-1010.

Gawryszewski, L. D. G., Riggio, L., Rizzolatti, G., \& Umiltà, C. (1987). Movements of attention in the three spatial dimensions and the meaning of "neutral" cues. Neuropsychologia, 25, 19-29.

Grove, P. M., KaneKo, H., \& ONO, H. (2001). The background inclination of a surface defined by empirical corresponding points. Perception, 30, 411-429.

HikosaKa, O., Miyauchi, S., \& Shimojo, S. (1993). Focal visual attention produces illusory temporal order and motion sensation. Vision Research, 33, 1219-1240.

HowARD, I. P., \& Rogers, B. J. (1995). Binocular vision and stereopsis. New York: Oxford University Press.

Howard, I. P., \& Rogers, B. J. (2002). Seeing in depth: Vol. 2. Depth perception. Toronto: Porteous.

Hung, G. K., Zhu, H., \& Ciuffreda, K. J. (1997). Convergence and divergence exhibit different response characteristics to symmetric stimuli. Vision Research, 37, 1197-1205.

ICHIKAWA, M. (2002, July). Visual simultaneity varies with the stimulus position in the visual field. Paper presented at the second Asian Conference on Vision, Gyeong ju, Korea.

ICHIKAWA, M., \& MASAKURA, Y. (2004). The connection of visual stimulus with observer's voluntary motion affects the flash-lag effect [Abstract]. Journal of Vision, 4(8), 581a.

ICHIKAWA, M., \& MASAKURA, Y. (2006). Manual control of the visual stimulus reduces the flash-lag effect. Vision Research, 46, 21922203.

Krekelberg, B., \& Lappe, M. (2001). Neuronal latencies and the position of moving objects. Trends in Neurosciences, 24, 335-339.

LANDERs, D. D., \& CoRMaCK, L. K. (1997). Asymmetries and errors in 
perception of depth from disparity suggest a multicomponent model of disparity processing. Perception \& Psychophysics, 59, 219-231.

LewIS, C. F., \& McBeath, M. K. (2004). Bias to experience approaching motion in a three-dimensional virtual environment. Perception, 33, 259-276.

LinARES, D., \& LóPEZ-Moliner, J. (2006). Perceptual asynchrony between color and motion with a single direction change. Journal of Vision, 6, 974-981.

López-Moliner, J., \& Linares, D. (2006). The flash-lag effect is reduced when the flash is perceived as a sensory consequence of our action. Vision Research, 46, 2122-2129.

Mitrani, L., SheKerdJiISKI, S., \& YakimofF, N. (1986). Mechanisms and asymmetries in visual perception of simultaneity and temporal order. Biological Cybernetics, 54, 159-165.

Miura, T., Shinohara, K., \& Kanda, K. (2002). Shift of attention in depth in a semi-realistic setting. Japanese Psychological Research, 44, 124-133.

Moutoussis, K., \& ZeKI, S. (1997a). A direct demonstration of perceptual asynchrony in vision. Proceedings of the Royal Society B, 264, 393-399.

Moutoussis, K., \& ZeKI, S. (1997b). Functional segregation and temporal hierarchy of the visual perceptive systems. Proceedings of the Royal Society B, 264, 1407-1414.

MuraKami, I. (2001). A flash-lag effect in random motion. Vision Research, 41, 3101-3119.

Mustillo, P. (1985). Binocular mechanisms mediating crossed and uncrossed stereopsis. Psychological Bulletin, 97, 187-201.

Nakamizo, S., Ono, H., \& Ujike, H. (1999). Subjective staircase: A multiple wallpaper illusion. Perception \& Psychophysics, 61, 13-22.

Nakayama, K. (1977). Human depth perception. Society of PhotoOptical Engineers, 120, 2-9.

Nijhawan, R. (1994). Motion extrapolation in catching. Nature, 370, 256-257.

NishidA, S., \& Johnston, A. (2002). Marker correspondence, not processing latency, determines temporal binding of visual attributes. Current Biology, 12, 359-368.

Patterson, R., Cayko, R., Short, G. L., Flanagan, R., Moe, L., TAYLOR, E., \& DAY, P. (1995). Temporal integration differences between crossed and uncrossed stereoscopic mechanisms. Perception \& Psychophysics, 57, 891-897.

Pisella, L., ArZi, M., \& Rossetti, Y. (1998). The timing of color and location processing in the motor context. Experimental Brain Research, 121, 270-276.

Portfors-Yeomans, C. V., \& Regan, D. (1996). Cyclopean discrimination thresholds for the direction and speed of motion in depth. Vision Research, 36, 3265-3279.

Posner, M. I., Snyder, C. R. R., \& Davidson, B. J. (1980). Attention and the detection of signals. Journal of Experimental Psychology: General, 109, 160-174.

Purushothaman, G., Patel, S. S., Bedell, H. E., \& Ogmen, H. (1998). Moving ahead through differential visual latency. Nature, 396, 424.

Rogers, B. J., \& ANSTIS, S. M. (1972). Intensity versus adaptation and the Pulfrich stereophenomenon. Vision Research, 12, 909-928.

Sheth, B. R., Nijhawan, R., \& Shimojo, S. (2000). Changing objects lead briefly flashed ones. Nature Neuroscience, 3, 489-495.

Shimojo, S., MiYauchi, S., \& HikosaKa, O. (1997). Visual motion sen- sation yielded by non-visually driven attention. Vision Research, $\mathbf{3 7}$, 1575-1580.

Shioiri, S., Yamamoto, K., \& Yaguchi, H. (2002). Effect of attention on flash lagging [Abstract]. Journal of Vision, 2(7), 27a.

Shirai, N., \& Yamaguchi, M. K. (2004). Asymmetry in the perception of motion-in-depth. Vision Research, 44, 1003-1011.

Stelmach, L. B., \& Herdman, C. M. (1991). Directed attention and perception of temporal order. Journal of Experimental Psychology: Human Perception \& Performance, 17, 539-550.

TAKEUCHI, T. (1997). Visual search of expansion and contraction. Vision Research, 37, 2083-2090.

TANaka, Y., \& Shimojo, S. (1996). Location vs feature: Reaction time reveals dissociation between two visual functions. Vision Research, 36, 2125-2140.

Tse, P. [U.], Cavanagh, P., \& Nakayama, K. (1998). The role of parsing in high-level motion processing. In T. Watanabe (Ed.), High-level motion processing: Computational, neurobiological, and psychophysical perspectives (pp. 249-266). Cambridge, MA: MIT Press.

Tse, P. U., \& Logothetis, N. K. (2002). The duration of 3-D form analysis in transformational apparent motion. Perception \& Psychophysics, 64, 244-265.

Viviani, P., \& Aymoz, C. (2001). Colour, form, and movement are not perceived simultaneously. Vision Research, 41, 2909-2918.

Whitney, D., \& MuraKami, I. (1998). Latency difference, not spatial extrapolation. Nature Neuroscience, 1, 656-657.

Woo, G. C., \& Sillanpaa, V. (1979). Absolute stereoscopic thresholds as measured by crossed and uncrossed disparities. American Journal of Optometry \& Physiological Optics, 56, 350-355.

YANG, Q., Bucci, M. P., \& KAPOULA, Z. (2002). The latency of saccades, vergence, and combined eye movements in children and in adults. Investigative Ophthalmology \& Visual Science, 43, 2939-2949.

\section{NOTE}

1. In the middle three depth position conditions in the large-disparity condition, the depth positions of the two stimuli are expected to correspond to the middle point between the two stimuli in the basic condition. For this reason, if the relationship between the disparity size and delay for each stimulus was fixed, the SOAs for the middle three depth position conditions could be predicted by averaging the summations of the two SOAs obtained in the basic condition. The SOAs for the aroundfixation-plane condition in the large-disparity condition can be predicted by averaging (1) the summation of the SOAs obtained for the in-frontof-fixation-plane condition and around-fixation-plane condition and (2) the summation of the SOAs obtained for the around-fixation-plane condition and behind-fixation-plane condition in the basic condition for each observer. The SOA for the in-front-of-fixation-plane (or behindfixation-plane) condition in the large-disparity condition can be predicted by averaging (1) the summation of the SOAs obtained for the nearest (or most distant) condition and in-front-of-fixation-plane (or behind-fixation-plane) condition, and (2) the summation of the SOAs obtained for the in-front-of-fixation-plane (or behind-fixation-plane) condition and around-fixation-plane condition in the basic condition.

(Manuscript received January 29, 2008; revision accepted for publication October 2, 2008.) 